\title{
Scalar Readout Model for the Super-Rens Focused Spot
}

\author{
Alberto C. Assafrao \\ a.dacostaassafrao@tudelft.nl \\ Silvania F. Pereira \\ H. Paul Urbach
}

\author{
Optics Research Group, Department of Imaging Science and Technology, Delft University of Technol- \\ ogy, Lorentzweg 1, 2628 CJ Delft, The Netherlands. \\ Optics Research Group, Department of Imaging Science and Technology, Delft University of Technol- \\ ogy, Lorentzweg 1, 2628 CJ Delft, The Netherlands. \\ Optics Research Group, Department of Imaging Science and Technology, Delft University of Technol- \\ ogy, Lorentzweg 1, 2628 CJ Delft, The Netherlands.
}

A scalar readout model is presented to investigate the readout characteristics of the super resolution near field disc. The super resolution effect is described by means of a threshold model, where the super resolution material imparts a phase change on the focused spot if the laser density energy is high enough to trigger the SRens effect. This approach results in a very fast way of computing the basic characteristics of the SRens readout signal, being suitable for large investigations. Moreover, many simulation results have been experimentally confirmed by other groups, which further validates the model. Thus, this simplified model is an useful tool for a better comprehension of the readout signal of the super resolution effect in optical data storage and other super resolution applications. [D0I: http://dx.doi.org/10.2971/jeos. 2011.11056]

Keywords: No keywords supplied

\section{INTRODUCTION}

With the recent Blu-Ray disc (BD) technology, optical data storage (ODS) has achieved its practical limit. Using a high numerical aperture $(\mathrm{NA}=0.85)$ lens and a short wavelength laser (405 nm), the BD system can resolve data marks as small as $138 \mathrm{~nm}$. Up to now, ODS has evolved by basically decreasing the wavelength of the light employed and increasing the focusing lens numerical aperture (NA), which allow the system to resolve smaller data marks. However, increasing the current ODS capacity by further reducing the wavelength employed or using higher NA lenses is no longer an option. For instance, we remark that the numerical aperture of a lens can reach a maximum value up to one (in air) and lasers operating at short wavelength can become expensive for commercial purposes. Thus, alternative techniques should be considered, if one wants to improve the current ODS system. Among those techniques, the super-resolution near-field effect (SRens) appears as a good candidate [1], [2]. In this technique, a thin layer of a non-linear material is placed on top of the data marks. The optical properties of the SRens layer change under laser radiation exposure, leading to a focused spot that is able to retrieve data information beyond the optical diffraction limit during the readout process [3]-[5]. It is believed that the underlying mechanism behind this change has to do with a thermally induced change of the super resolution material from a solid to a molten state [6]-[10]. In spite of all experimental work done in this subject, not much literature regarding the readout mechanism is found [11],[12],[13]. In any case, to model such an effect, rigorous simulation tools are desirable. However, for a complete simulation tool that computes the readout spot out of a SRens disc, one has to rigor- ously calculate the incident focused spot through a multilayer structure, including the optically inhomogeneous non-linear layer, where the thermal effect takes place. Then, the interaction of the focused spot with the data structure must be calculated and the scattered field has to be back propagated to the far field, after passing throughout the whole multilayer stack. Finally, the far field distribution must be integrated over the pupil lens, which leads to the intensity on the detector. Using the procedure above, one will only get the detected intensity of one single position on the data disc. If the full readout signal is to be obtained, let us say, over few data marks in a row, the whole calculation has to be repeated again on different disc positions. Moreover, if one wants to study the influence of adjacent data tracks on the readout signal, the so-called crosstalk effect, the number of simulations needed increases dramatically, becoming prohibitive large. Therefore, simplified models for the SRens problem must be employed.

In this paper, we propose a scalar model that incorporates the SRens effect on the focused spot, using a threshold based model, and retrieves the readout signal out of a SRens optical disc. We have simulated the basic aspects of the SRens readout signal and applied the model to investigate the crosstalk effect in a SRens disc. In addition, we have studied a two beam crosstalk cancellation scheme that takes advantage of the SRens focused spot features [14]. The algorithm implementation is easy, the execution is very fast and the results achieved are qualitatively in agreement with experiments. Furthermore, for large optimizations and crosstalk analysis, this tool becomes very useful. Although we have applied this 
model for analyzing the Digital Versatile Disc (DVD) system, it could also be used for BD system by proper rescaling of the optical parameters. Nevertheless, we emphasize that the main predictions from this model should be checked at some point with a more rigorous vectorial model.

This paper is organized as follows: in $§ 2$ we present details of the scalar readout theory. In $\$ 3$ we show how the SRens threshold model is incorporated in the focused spot and in $\S 4$ we present simulation results using the method. In section $\S 5$ we present the scalar explanation for the modulated readout signal beyond the diffraction limit. $\S 6$ is dedicated to crosstalk analysis, including a two-beam crosstalk cancellation scheme and an estimate of how much the distance between adjacent tracks can be reduced in a SRens disc. Finally we give the conclusions of this work in $\S 7$.

\section{Scalar Diffraction Theory of Read Out Systems}

In what follows, we briefly review the scalar theory of readout systems. For further details, we refer to Refs. [15]-[18].

\subsection{Fast Fourier Transform Approach}

In Fig. 1 we show the lightpath of an optical data storage system (shown in transmission). The incident light distribution $f(x, y)$ is focused onto the disc by the objective lens. Apart from a scaling factor and a phase term, the field in focus is given by

$$
F(u, v)=\int_{-\infty}^{+\infty} \int_{-\infty}^{+\infty} f(x, y) \exp [-2 \pi i(u x+v y)] d x d y
$$

where $(u, v)$ are the coordinates in the image plane, normalized by $\lambda / \mathrm{NA}$, and $(x, y)$ are the coordinates in the pupil plane. The focused field distribution $F(u, v)$ interacts with the data marks, described by a reflection function $R(u, v)$. The product $U_{T}(u, v)=F(u, v) \cdot R(u, v)$ is propagated to the collector lens according to

$$
\begin{aligned}
g(x, y)=\int_{-\infty}^{+\infty} \int_{-\infty}^{+\infty} & F(u, v) R(u, v) \\
& \times \exp [-2 \pi i(u x+v y)] d u d v
\end{aligned}
$$

The field distribution $g(x, y)$ is then integrated over the pupil area, resulting in the final intensity on the detector of a given position, after computing $|g(x, y)|^{2}$. The modulated readout signal is obtained by moving the disc data marks underneath the focused spot and computing Eq. 2 for many translated positions.

\subsection{Reflected field decomposition}

The scalar readout program can be further upgraded by decomposing the optical disc components [18]. The idea behind this approach is to decompose the light reflected from the disc into separate components and, after performing the linear diffraction operation (Fourier transform), recombine them. The result is a detailed description of the readout process, especially useful for crosstalk analysis. Suppose that the field

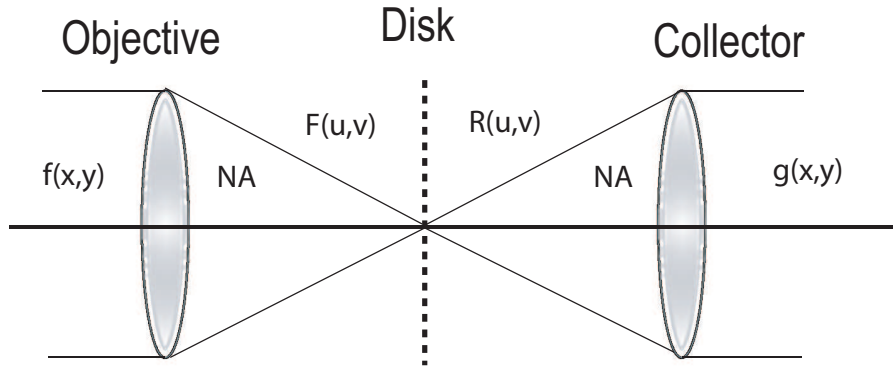

FIG. 1 Lightpath for a scalar readout program. The input field distribution $f(x, y)$ is propagated to the disc plane by means of a Fourier transform resulting in the field distribution $F(u, v)$ that interacts with the disc reflection function $R(u, v)$. The product $U_{T}(u, v)=F(u, v) \cdot R(u, v)$ is back propagated to the collector lens, resulting in the field distribution $\mathrm{g}(\mathrm{x}, \mathrm{y})$. Here we show a readout system in transmission.

reflected by the disc is given by $U_{T}(u, v)=F(u, v) \cdot R(u, v)$. Since the model is linear in the reflection function, we can rewrite the total field reflected from the disc as the summation of the fields reflected from all separate areas, i.e.,

$$
U_{T}(u, v)=\sum_{m=1}^{N} U_{m}(u, v)
$$

where $U_{m}(u, v)$ are due to reflection from areas on the disc that do not overlap. By adding up all components, the whole disc is uniquely described. If one applies a linear operator on $U_{T}(u, v)$, the operation can be performed on each components individually, i.e.,

$$
\hat{U}_{T}(u, v)=\sum_{m=1}^{N} \hat{U}_{m}(u, v)
$$

where the hat represents a linear operation, e.g. the Fourier transform. In Fig. 2 we show the decomposition of a disc reflection function. The track being scanned is described by $U_{M 1}$ and the surrounding track is $U_{M 2}$. The reflection from the land is $U_{L}$. Thus, we write the total reflection $U_{T}$ as,

$$
U_{T}=r_{L} U_{F}+\left(r_{M}-r_{L}\right)\left(U_{M 1}+U_{M 2}\right)
$$

where $r_{L}$ is the complex reflectance of the land, $r_{M}$ is the complex reflectance of the marks and $U_{F}$ is the reflection from a flat and uniform surface. The components $U_{M 1}$ and $U_{M 2}$ can assume only values of zero and one. To include groove structures in the calculation, one has only to change the flat field reflection term to $U_{F}=\exp [i \phi(u, v)]$, where $\phi(u, v)$ represents the phase imparted to the reflection field as function of position.

\subsection{Generation of signal, servo and crosstalk terms}

To compute the readout signal from this approach, we remind that the field intensity falling on the detector is proportional to $\left|\hat{U}_{T}\right|^{2}$, where $U_{T}$ is given by equation 5 and the hat represents the Fourier transform of $U_{T}$. Thus,

$$
\left|\hat{U}_{T}\right|^{2} \approx\left|r_{L} \hat{U}_{F}\right|^{2}+\left|\Delta r \hat{U}_{M 1}\right|^{2}+\left|\Delta r \hat{U}_{M 2}\right|^{2}+
$$




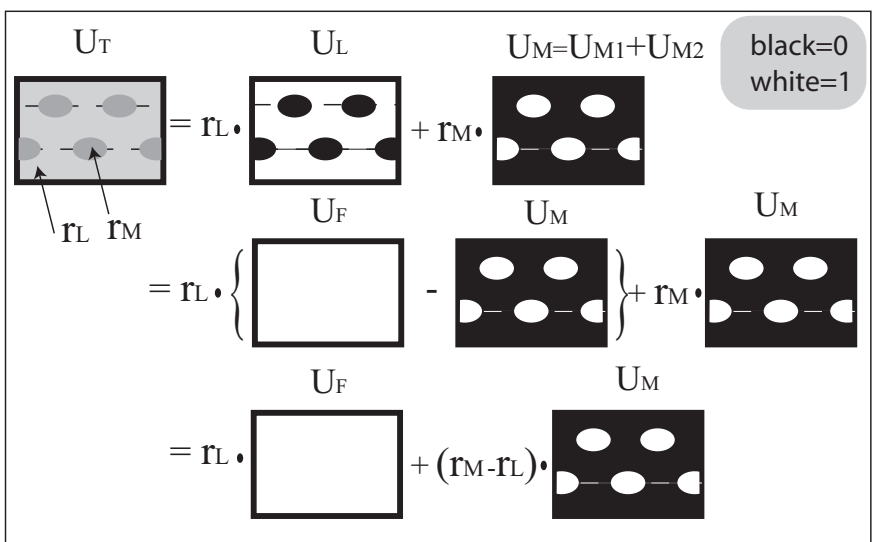

FIC. 2 Decomposition of the field reflected from the disc. All field components that come from the patterns $U_{M 1}, U_{M 2}$ and from the flat media $U_{F}$ are calculated separately being linearly added to produce the final readout signal.

\begin{tabular}{|l|c|l|}
\hline Term name & Formula & Definition \\
\hline \hline Background & $\left|r_{L} \hat{U}_{F}\right|^{2}$ & $\begin{array}{l}\text { Background } \\
\text { reflectance }\end{array}$ \\
\hline Basic data & $\left|\Delta r \hat{U}_{M 1}\right|^{2}$ & $\begin{array}{l}\text { on/on track } \\
\text { interference }\end{array}$ \\
\hline $\begin{array}{l}\text { Type 0 } \\
\text { crosstalk }\end{array}$ & $\left|\Delta r \hat{U}_{M 2}\right|^{2}$ & $\begin{array}{l}\text { off/off track } \\
\text { interference }\end{array}$ \\
\hline Servo & $2\left|r_{L} \hat{U}_{F} \Delta r \hat{U}_{M 1}\right| \cos \Phi_{F_{1}}$ & $\begin{array}{l}\text { on track/background } \\
\text { interference }\end{array}$ \\
\hline $\begin{array}{l}\text { Type 1 } \\
\text { crosstalk }\end{array}$ & $2\left|r_{L} \hat{U}_{F} \Delta r \hat{U}_{M 2}\right| \cos \Phi_{F_{2}}$ & $\begin{array}{l}\text { off track/background } \\
\text { interference }\end{array}$ \\
\hline $\begin{array}{l}\text { Type 2 } \\
\text { crosstalk }\end{array}$ & $2\left|\Delta r^{2} \hat{U}_{M 1} \hat{U}_{M 2}\right| \cos \Phi_{12}$ & $\begin{array}{l}\text { on/off track } \\
\text { interference }\end{array}$ \\
\hline \hline
\end{tabular}

TABLE 1 Independent terms resulting from Babinet decomposition.

plus the crossed terms

$$
\begin{aligned}
& +2\left|r_{L} \hat{U}_{F} \Delta r \hat{U}_{M 1}\right| \cos \left(\phi_{r_{L}}+\phi_{U_{F}}-\phi_{\Delta_{r}}-\phi_{U_{M 1}}\right)+ \\
& +2\left|r_{L} \hat{U}_{F} \Delta r \hat{U}_{M 2}\right| \cos \left(\phi_{r_{L}}+\phi_{U_{F}}-\phi_{\Delta_{r}}-\phi_{U_{M 2}}\right)+ \\
& +2\left|\Delta r^{2} \hat{U}_{M 1} \hat{U}_{M 2}\right| \cos \left(\phi_{U_{M 1}}-\phi_{U_{M 2}}\right)
\end{aligned}
$$

Each of these terms is related to a specific type of signal that together form the total readout signal. For instance, diffraction from the flat surface $\left|r_{L} \hat{U}_{F}\right|^{2}$ forms the background signal, $\left|\Delta r \hat{U}_{M 1}\right|^{2}$ is the basic data, the term $\left|\Delta r \hat{U}_{M 2}\right|^{2}$ describes the interference of the off-track mark pattern with itself (called type 0 crosstalk). The cross terms have the following interpretation: $2\left|r_{L} \hat{U}_{F} \Delta r \hat{U}_{M 1}\right| \cos \Phi_{F_{1}}$, where $\Delta r=r_{M}-r_{L}$ and $\Phi_{F 1}=\left(\phi_{r_{L}}+\phi_{U_{F}}-\phi_{\Delta_{r}}-\phi_{U_{M 1}}\right)$, is the interference of the on-track mark pattern with the background (servo signal), $2\left|r_{L} \hat{U}_{F} \Delta r \hat{U}_{M 2}\right| \cos \Phi_{F 2}$ is the interference of the off-track mark pattern with the background (type 1 crosstalk) and $2\left|\Delta r^{2} \hat{U}_{M 1} \hat{U}_{M 2}\right| \cos \Phi_{12}$ is the interference of the on-track and the off-track mark patterns (type 2 crosstalk). In all terms, $\Phi_{F_{1}}$, $\Phi_{F_{2}}$ and $\Phi_{12}$ are the phase difference between the components as function of position in the pupil. A summary of the terms resulting from the expansion is listed in the Table 1.

\section{Scalar threshold model for SRens}

We will now consider the super resolution effect. A schematic view of the super resolution effect is shown in the Fig. 3a. As the focused spot passes through the layer stack, which is usu-

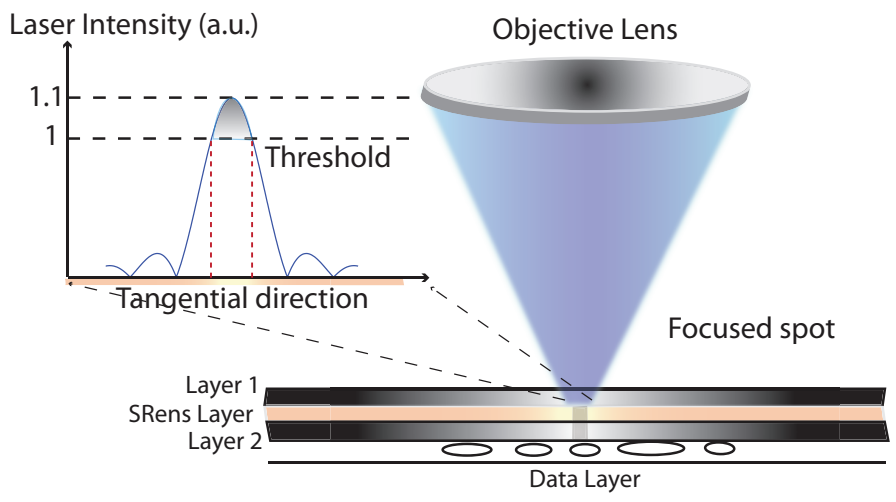

(a) Super resolution effect

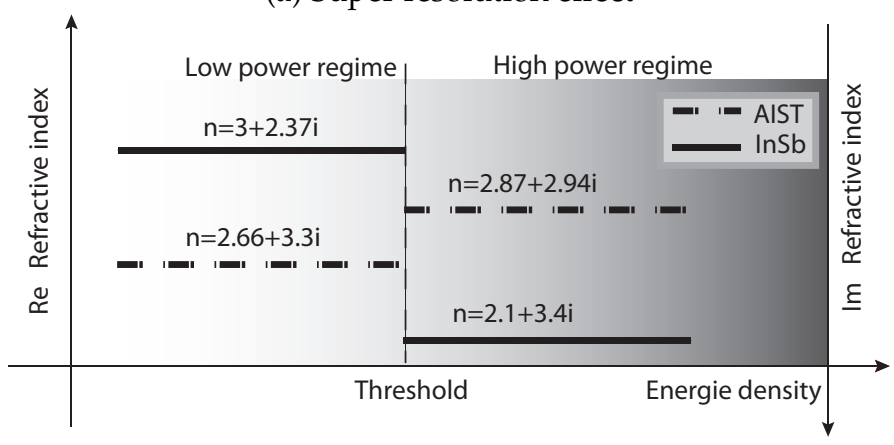

(b) Refractive index of SR materials

FIG. 3 Schematics of the SRens effect and materials used. a) The focused spot incident on SR layer stack opens an optical aperture if the intensity is high enough to trigger the nonlinear effect. b) Refractive index of two materials which shows a nonlinear behavior on its optical properties as function of the field intensity.

ally composed of a super resolution material sandwiched by two layers of $\mathrm{ZnS}: \mathrm{SiO}_{2}$, there will be a local change in the optical properties of the super resolution layer when the laser energy density is enough to trigger the nonlinear effect [19]. Suppose that initially the material is in the solid state, having a certain refractive index $n_{1}$. When the laser spot is focused onto the super resolution layer, then in the portion of the spot where the electric energy density is above the material super resolution threshold, the material will melt, leading to a fast change in the refractive index from $n_{1}$ to $n_{2}$. Note that the complex part of the refractive index of the molten region may become higher (or lower) than it was in the solid state. For this reason, the molten region can be regarded as a scatterer-like structure or an aperture like structure. However, in either case, the general name 'optical aperture' is given for the molten region. A summary of the refractive indexes values of the two materials studied in this work, Indium Antimonite (InSb - scatterer like) and AgInSbTe (AIST - aperture like), is given in the Fig. 3b. [20]-[23]

In our model we assume that the phase change occurs instantaneously. The super resolution effect is then incorporated into the focused beam by a routine that searches the intensity values in the field distribution which are above or below a given threshold. We define this threshold as 1 , in an arbitrary unit system. The effect of the super resolution layer is imparting an amplitude and a phase change on the focused field, according to

$$
\Phi_{S R}(u, v)=\exp [i k z n(u, v)]
$$

where $k=2 \pi / \lambda, \lambda$ the wavelength of the light employed, $z$ 


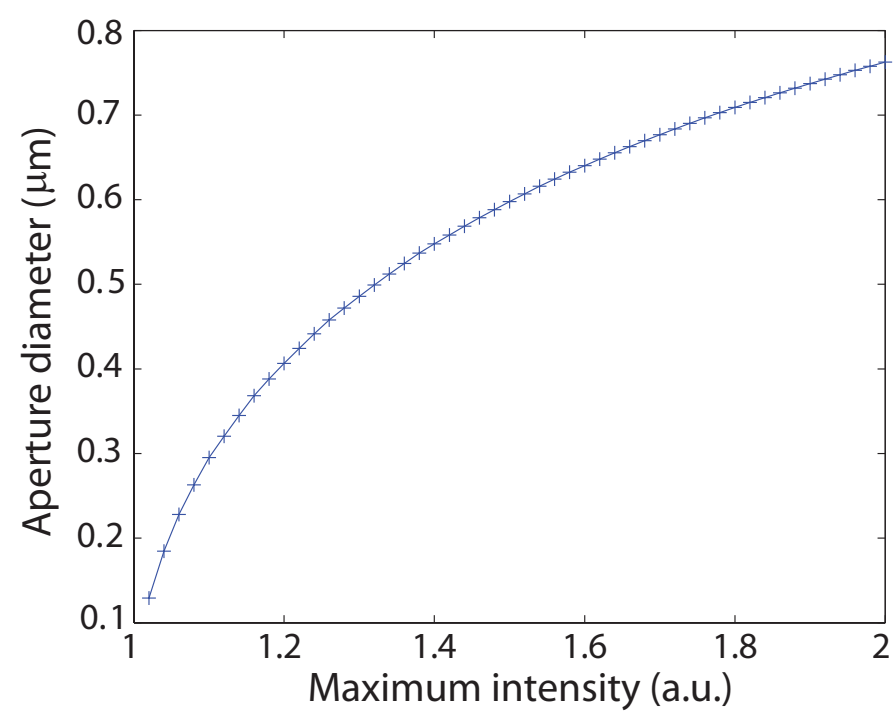

FIG. 4 Optical aperture diameter generated by the focused Airy spot as function of the laser power.

is the layer thickness and $n$ is the (usually complex) refractive index, which can be either before $\left(n_{b}\right)$ or after $\left(n_{a}\right)$ the threshold. Thus, if the focused spot intensity $\left(|F(u, v)|^{2}\right)$ value in a given position $\left(u_{1}, v_{1}\right)$ is higher than the threshold, the effect of the super resolution layer will be a phase change in the form of $E_{S R}=F\left(u_{1}, v_{1}\right) \cdot \exp \left(i k z n_{a}\right)$ at that position.

Based on the algorithm described above, we can compute the diameter of the molten region as function of the laser power by determining the area of the focused spot which has the energy density above the threshold value. The result is plotted in the Fig. 4.

In Figs. 5a to 5c, we show the focused spots before and after applying the super resolution algorithm, for a system with $\lambda=650 \mathrm{~nm}$ and NA $=0.6$. When the super resolution effect is not activated, the focused spot has the typical Airy pattern distribution, as shown in Fig. 5a. If the InSb layer is present and activated (laser intensity higher than the threshold), the focused spot becomes slightly broader at the full width half maximum (FWHM) and presents a sharp structure near the top. In the case of AIST layer, the focused spot becomes narrower and the side lobes are smaller in comparison to the other spots. Note that the maximum intensity value of the SRens focused spot decreases due to the absorption in the super resolution layer. For these calculations, we assumed an optical aperture of $300 \mathrm{~nm}$ radius (i.e. peak intensity of 1.1 before the SRens layer, see Fig. 3a). The field distribution profiles along the tangential direction are shown in the Fig. $5 \mathrm{~d}$ and a normalized profile, zoomed at the top, is shown in the Fig. 5e. In all cases, the scalar approach gives a focused spot that resembles those computed with rigorous simulations [24]. Moreover, the SRens effect algorithm takes less than 1 second to be executed, while rigorous simulations can take up to 5 hours.

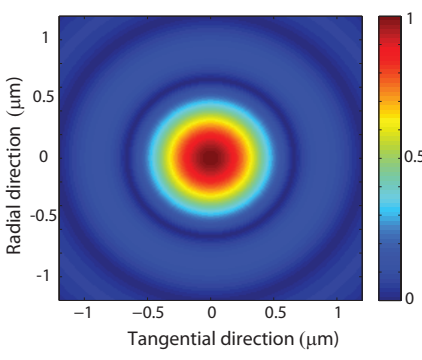

(a) SR off

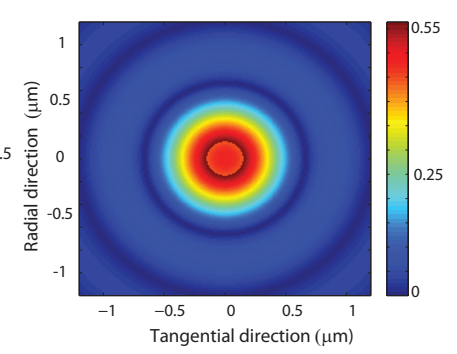

(b) InSb

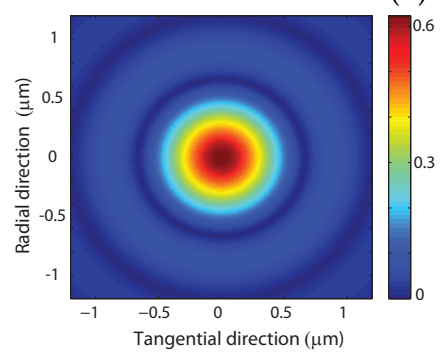

(c) AIST

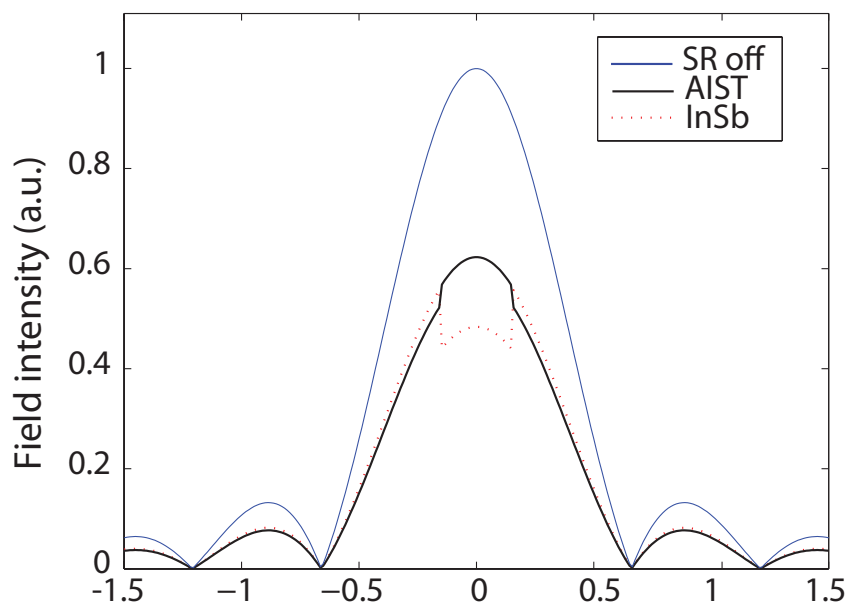

Tangential direction ( $\mu \mathrm{m})$

(d) Spot Profile

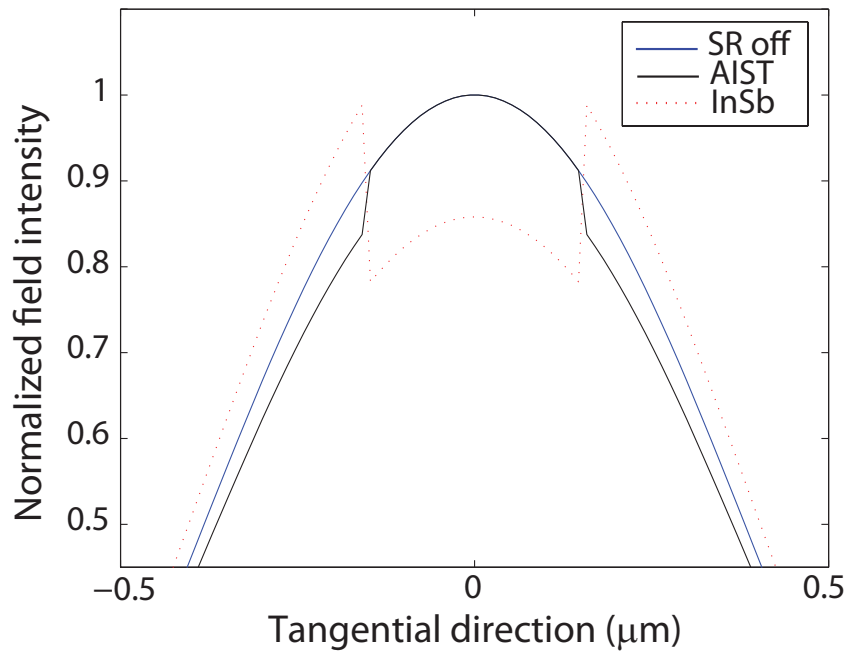

(e) Spot profile (zoom at top)

FIC. 5 The focused spot after the super resolution effect. a) to c) Focused spot without the SRens effect, with the InSb and AIST super resolution layers, respectively. d) The intensity profile along the tangential direction. e) Zoom at the top of d), after all distributions are normalized to unity. 


\section{Readout of a super resolution beam}

In the previous section we described an algorithm that incorporates the super resolution effect on the focused spot using the scalar approach. Now we will use the super resolution spot on several disc structures to study its effect on the readout signal.

\subsection{On the computational effort}

As mentioned before, the SRens effect is incorporated in the focused spot without any computational effort. However, for the disc readout, the computation of the equation 2, performed via Matlab FFT built-in function, dictates how fast the simulations will be done. In a typical simulation section, one can define a 20 by $20 \mu \mathrm{m}^{2}$ computational window, divided into 1024 number of points, which results in a grid with resolution of $20 \mathrm{~nm}$. Under this assumptions, the FFT computation, including the time necessary to move the underneath data mask, will take around 1 second, in a home computer Pentium D, 2.8GHz with 4GB RAM. Thus, scanning one period of a periodic data structure with 1000nm length, with resolution of 20nm and considering the SRens effect, will last no more than 1 minute. Nevertheless, better accuracy can be achieved by increasing the number of points. Throughout this work, we have considered 1800 number of points, resulting in a mesh grid with resolution of $11 \mathrm{~nm}$.

\subsection{Readout system operating in reflection}

In our first analysis, we are interested in investigating how the visibility curve is affected by the SRens effect. The visibility curve is calculated from the modulated readout signal of a periodic structure, being defined as $\left(I_{\max }-I_{\min }\right) /\left(I_{\max }+I_{\min }\right)$, usually plotted versus the data mark size. Therefore, the visibility curve is a good indication of the system resolution. We first consider the system operating in reflection. This means that the reflected field from the disc surface has to pass again through the SRens layer, where the optical aperture remained open during the reflection process. We used a phase change disc with land reflectivity $r_{L}=0.9$ and mark reflectivity $r_{M}=0.002$ along with DVD optical parameters $(\lambda=650 \mathrm{~nm}$ and $\mathrm{NA}=0.6)$. The data marks were arranged periodicaly with duty cycle of $50 \%$. The results are shown in the Figs. $6 \mathrm{a}$ and $6 \mathrm{~b}$.When the super resolution is not activated, the smallest detectable period is given by the diffraction limit, $\lambda / 2 \mathrm{NA}=540 \mathrm{~nm}$ (data mark of 270nm). In direct comparison, the visibility curves computed with the AIST spot show an improvement in all frequencies, for all readout powers. However, when the laser intensity is too high (green curve tagged with diamonds), the visibility curve becomes similar to the one computed without SRens (blue curve). The reason is that the big optical aperture created at such power which does not influence significantly the focused spot shape. For the InSb readout, on the other hand, a huge improvement on the visibility is noticed at frequencies beyond the diffraction limit. However, at low frequencies, the visibility is smalller when compared to the readout without SRens. Although super resolution is achieved for both cases (visibility beyond the diffrac-

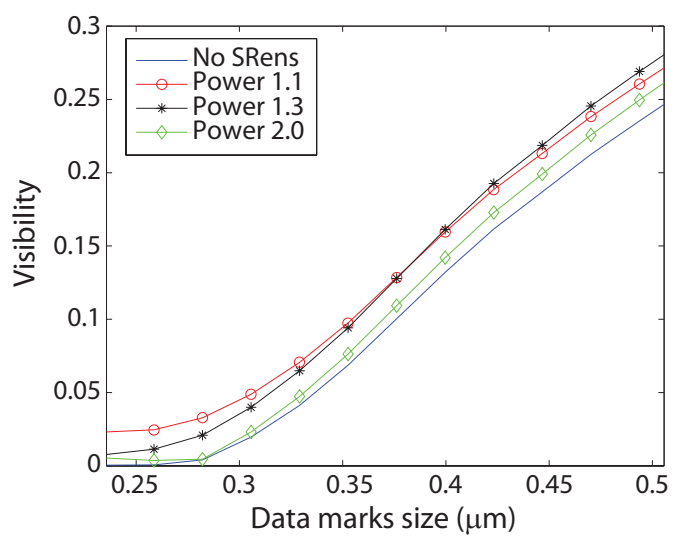

(a) AIST visibility curve

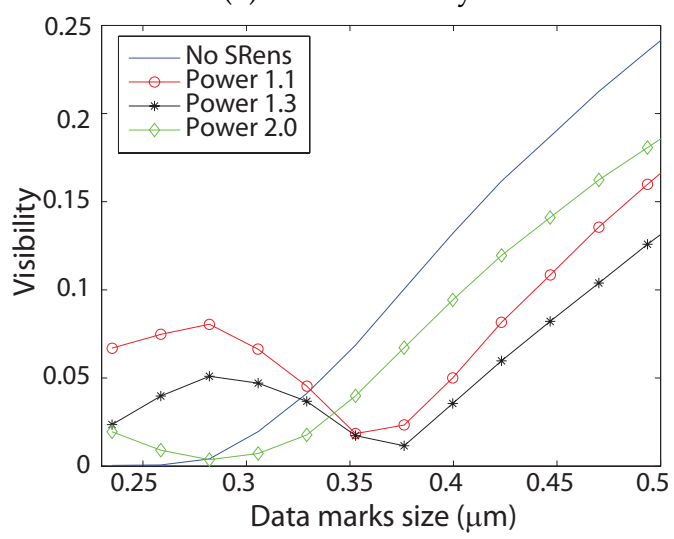

(b) InSb visibility curve

FIC. 6 Visibility curve as function of the data marks length for different readout power for the system operating in reflection. When the SRens is off, the smallest detectable mark has length given by $\lambda / 4 \mathrm{NA}=270 \mathrm{~nm}$. (a) The AIST readout spot shows a better resolution than the normal beam for all frequencies. (b) For the InSb spot readout, a better resolution at frequencies beyond the diffraction limit is verified, but at the cost of losses at low frequencies.

tion limit), in the InSb readout it comes at cost of a loss in the signal at some specific spatial frequency (330 $\mathrm{mm}$ for the 1.1 readout power), as suggested by the plots. This is particularly dangerous for improving the recording density, because the shortest marks in current DVD discs are $400 \mathrm{~nm}$. In this sense, the InSb will not improve the recording density. Experimental evidences of low frequency signal losses for SRens effect can be found in the reference. [25]

\subsection{Readout system operating in transmission}

We repeated the visibility simulation for the system operating in transmission. In the Figs. $7 \mathrm{a}$ and $7 \mathrm{~b}$ we show the resolution curve for the InSb and AIST materials considering different laser powers. Generally, the visibility curve behavior computed for both materials did not differ significantly from the ones presented in the reflection mode, though the absolute values are smaller. Since we are interested in the qualitative behavior of the SRens readout signal, we will proceed with our analysis using the transmission mode. Hence, results can be better interpretated and the field decomposition technique presented in the previous section can be applied without constraints. 


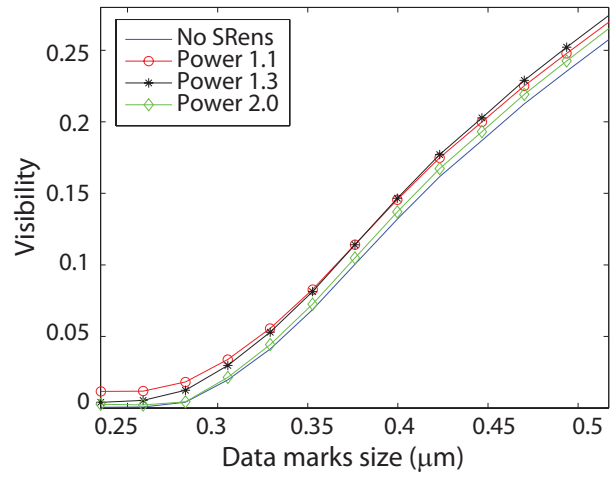

(a) AIST visibility curve

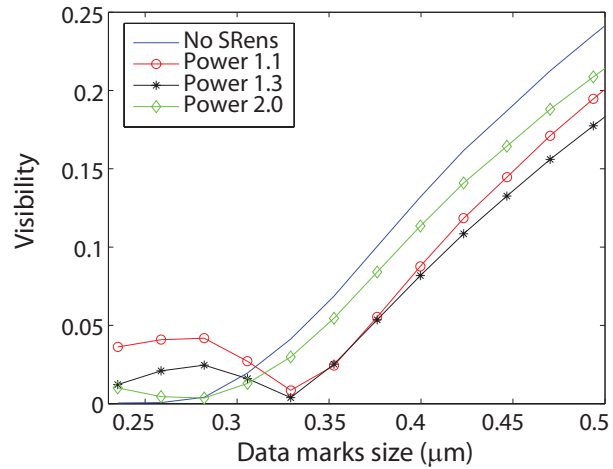

(b) InSb visibility curve

FIG. 7 Visibility curve as function of the data marks size for different readout power for the system operating in transmission. The general behavior of the curves remains the same as in reflection.

The readout signal of each individual data mark used to obtain the curves $7 \mathrm{a}$ and $7 \mathrm{~b}$, are plotted together as function of scan position in Figs. 8a (AIST) and 8b (InSb), respectivelly, for the laser maximum intensity at 1.1. Each curve shows the modulated signal obtained for a specific data mark size, in a periodic structure with duty cycle of $50 \%$, starting the scanning from the center of the mark and ending after one entire period is read. For example, in Fig. 8a, the blue curve tagged with * shows the AIST readout of a data mark with $517 \mathrm{~nm}$ length. The modulated signal varies from 0.483 up to 0.839 (visibility of 0.269 ), reaching the maximum when the readout spot is on the middle of the land. As the data marks become smaller, the amplitude of the modulated signal becomes also smaller, eventually reaching a point where no modulation is observed. Note that the modulated signal retains its shape for each data mark considered. For the InSb, however, the modulated signal shows an inversion between data marks smaller than $330 \mathrm{~nm}$ (see $8 \mathrm{~b}$ ). The maximum of the modulated signal for data marks with size bigger than $330 \mathrm{~nm}$ occurs when the readout spot is on the middle of the land while, for marks smaller then $330 \mathrm{~nm}$, the maximum occurs when the readout spot is on the middle of the mark. As the readout signal from the low frequency to high frequency tends to invert its modulation, there will be one point where the amplitude in the modulation signal is nearly zero. This point corresponds to the dip shown in Fig. $7 b$. Similar results are experimentally achieved in the references [20] and [25].

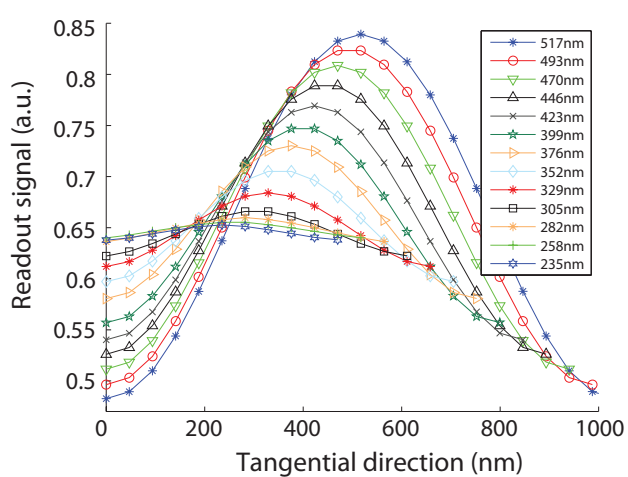

(a) AIST readout

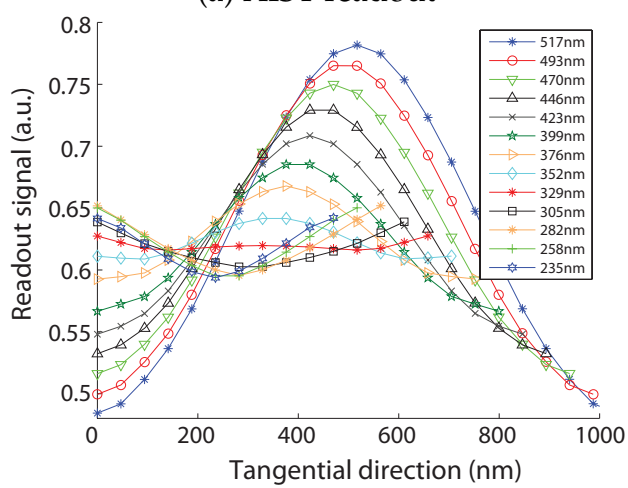

(b) InSb readout

FIG. 8 Modulated readout signal of individual marks for AIST and InSb spots. a) The modulated readout signal for the AIST spot keeps the same shape for every data mark. b) For the InSb spot, an inversion on the modulated signal is observed for data marks smaller than $330 \mathrm{~nm}$.

\subsection{Pit depth dependence}

It is well known that the pit depth affects the modulated readout signal and it has been shown that the best modulation occurs for a pit depth of $0.25 \lambda$ [18]. We will now investigate if the same holds for the SRens readout spot. In the Fig. 9a, we show the visibility computed as function of pit depth for a disc containing a data mark with $500 \mathrm{~nm}$ length. When the super resolution is off, the visibility is indeed maximized at pit depth of $0.25 \lambda$. If the AIST super resolution spot is activated, the visibility is slightly increased and the maximum still occurs at pit depth $=0.25 \lambda$. When the InSb readout spot is activated, a small shift in the visibility towards deeper pit depth is observed. This suggests that, for InSb readout spot, the ideal pit depth is related to the pit size. To investigate this possibility, we computed the same curve using data marks with $330 \mathrm{~nm}$ length. As shown in the Fig. 9b, the AIST visibility behavior is slightly changed by the pit depth while the visibility of the readout spot without SRens effect remains unaltered. On other hand, the behavior of the InSb visibility completely differs from the one calculated for $500 \mathrm{~nm}$. Simulation shows that the best modulation occurs if the pit depth is $0.35 \lambda$. This is an indication that the pit depth can be optimized for the InSb spot to give the best readout signal at each data mark frequency. In all simulations, we considered a ROM type of disc, with land reflectivity of $r_{L}=0.9$ and the mark reflectivity of $r_{M}=0.9 \exp (2 i k \cdot d e p t h)$, and the readout intensity was normalized by the signal computed when no marks were present. 


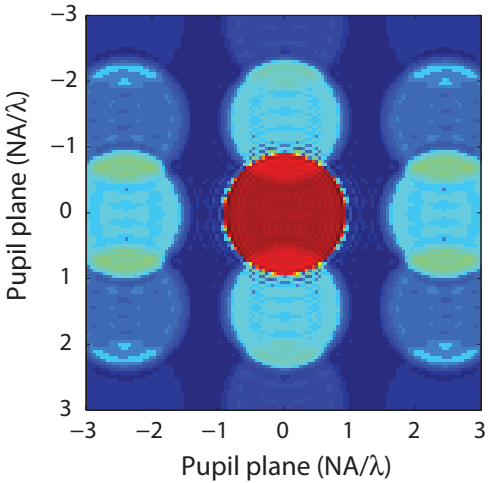

(a) SR off

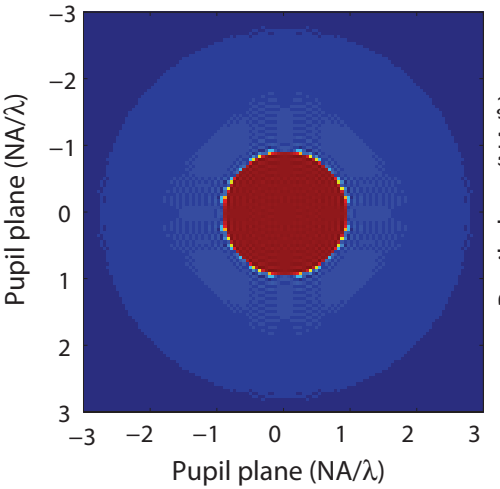

(b) SR on - Flat disc

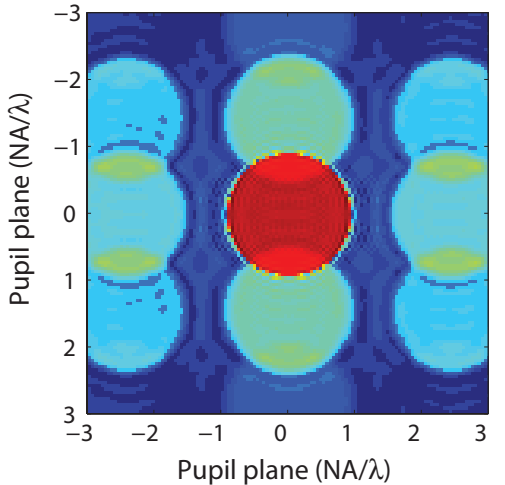

(c) SR on - Data disc

FIG. 10 Diffracted orders on the pupil plane. a) Beyond the cut-off frequency, no overlapping of the \pm 1 st orders with the oth order occurs for a focused spot without SRens. b) The SRens spot reading a disc without any marks on it generates a enlarged oth order in the lens pupil. c) The interference patterns between orders suggests that the \pm 1 st diffracted orders are also enlarged, which allows overlapping even beyond the cut-off frequency.

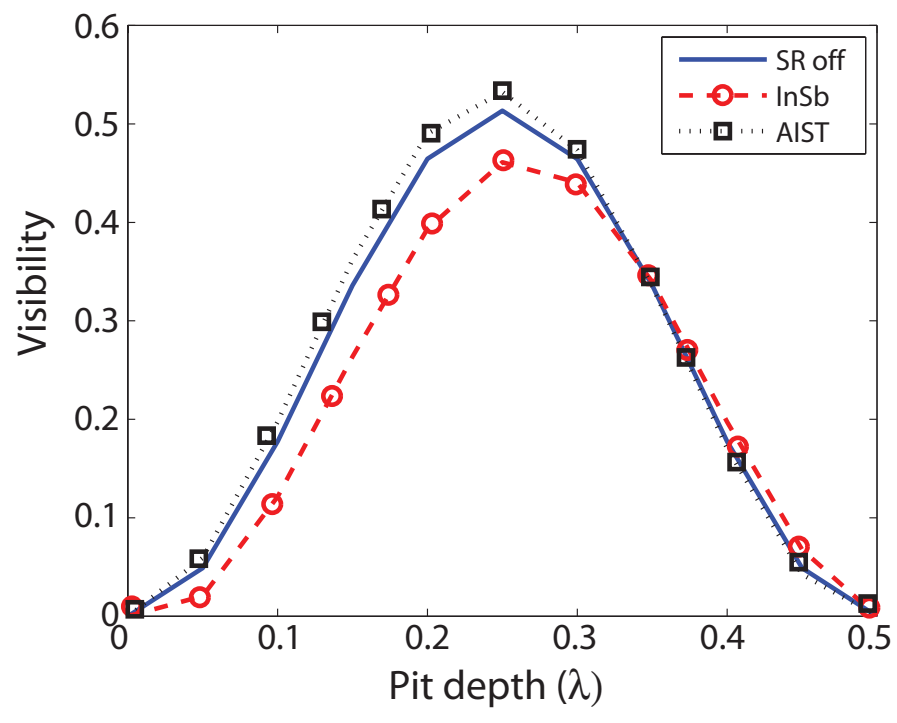

(a) 500 nm mark size

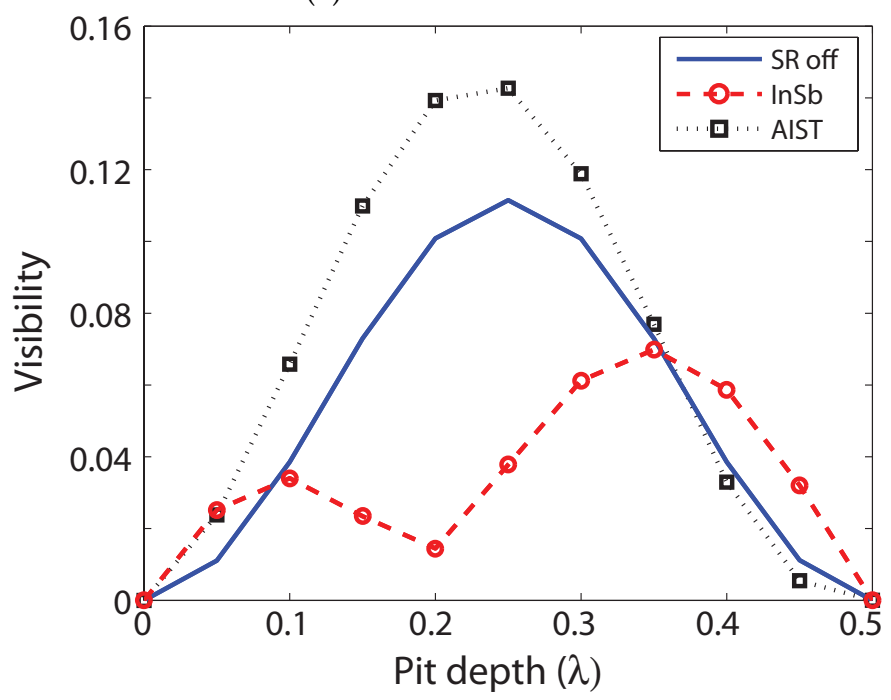

(b) 330nm mark size

FIG. 9 Visibility as function of the pit depth. (a) the best visibility for the readout spot without SRens and the AIST spot occurs at $0.15 \lambda$. (b) For $330 \mathrm{~nm}$ data marks, the best visibility occurs when the pit depth is $0.35 \lambda$ for the InSb spot.

\section{An interpretation for the readout signal beyond the diffraction limit.}

Although modulated signals beyond the diffraction limit have been experimentally observed during AIST or InSb readout, the general readout mechanism that leads to it has not been clarified. The scalar model gives us an useful interpretation on how the modulated signal at high frequencies is obtained. Let us first consider a periodic structure being read by a spot without super resolution effect. By evaluating and plotting Eq. 2, we find the intensity distribution of the scattered field on the pupil plane. According to the general scalar readout theory, if part of the \pm 1 st diffracted orders falls inside the zeroth order, there will be modulation in the readout signal. When the period of the marks becomes smaller, the overlap of the 0th and the \pm 1 st orders becomes less and hence the amplitude of the modulated signal diminishes. At a given frequency, there will be no overlap between orders and the result is no modulation in the readout signal. This frequency is known as the cut-off frequency. As an illustration, we plot in the Fig. 10a the intensity distribution in the pupil plane for a periodic disc with periodicity of 400nm (beyond the cut-off frequency) and duty cycle of $50 \%$. The adjacent traks are $700 \mathrm{~nm}$ far from the scanned track. As seen, the first orders are not overlapping with the zeroth one in the tangential direction, and therefore no modulation in the readout signal can be expected. At this point, we remind that the zeroth order (the red circle) corresponds, in fact, to the collector lens pupil. Next, let us consider a flat disc (without any data marks on it) and a focused spot with InSb SRens effect activated. By computing the intensity distribution in the pupil plane, we will find that the 0th order is enlarged in diameter by a factor of three (see Fig. 10b). Please notice that even though the zeroth order is enlarged, the lens pupil size remains unaltered, always in the range -1 to 1 , in diffration units. Finally, we compute the intensity distribution on the pupil plane generated by the InSb SRens spot reading the same periodic structure as before, as shown in the Fig. 10c. Here, we can clearly observe interference signals in between the diffracted orders. This suggests, in fact, that not only the 0th order was enlarged, but also the \pm 1 st orders. As the result, it becomes possible to have constructive/destructive interference of these enlarged \pm 1 st diffracted orders within the lens pupil 
(red circle), even for data marks with frequencies beyond the cut-off frequency. In this way, we achieve super resolution readout signal.

\section{Crosstalk analysis on SRens disc.}

In the previous sections we have described the threshold model based on the scalar theory and simulated the readout signal of some cases of interest. In this section, we will use all developed tools to investigate the crosstalk effect in optical discs in the presence of the super resolution effect. Generally speaking, crosstalk is caused by the undesired signal from data marks on adjacent tracks, which distorts the readout signal generated from the data being read. In particular, we are interested in investigating the crosstalk cancelation method based on grooved-land discs and we discuss the possibility of a two-beam crosstalk cancellation scheme where the super resolution readout spot is used to our advantage.

In Table 1 we showed all independent terms resulting from the decomposition of the disc reflection function [26]. These independent terms can be combined to form meaningful signal groups to describe the crosstalk problem, as shown in Fig. 11. By combining the background, data and the servo signals, we form the base signal, that is responsible for generating the total current from the scanned track with data mark on it. The single modulation crosstalk (SMX) is formed by the type0 crosstalk and type1 crosstalk, and generates the current that results from a track that contains marks and is adjacent to the empty scan track. Finally, the inter modulation crosstalk (IMX) results from the interference between the data marks on the scanned track and the data marks on an adjacent track.

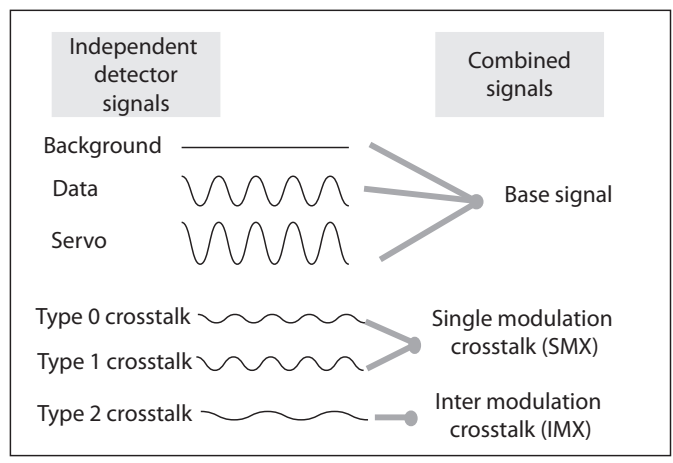

FIG. 11 Combination of independent detector signals formed from the linear disc decomposition.

\subsection{Grooved-land SRens disc.}

In a disc containing groove and land structure, it has been shown that an optimal crosstalk cancellation occurs when the groove depth is around $0.15 \lambda$ [27]. It is interesting to investigate if the crosstalk levels of a SRens readout spot for a grooved-land disc can be also minimized in the same way. In the Figs. 12a and $12 \mathrm{~b}$ we show the single modulation crosstalk and the inter modulation crosstalk signals for a flat

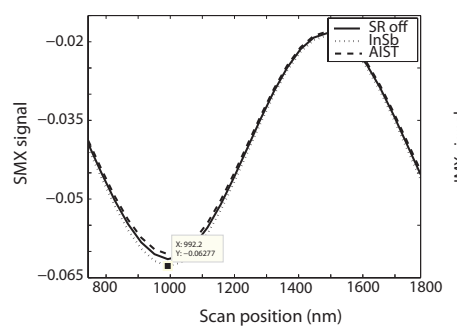

(a) Flat media SMX

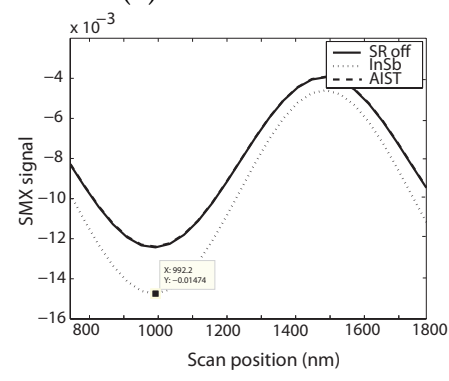

(c) Groove depth $=0.15 \lambda \mathrm{SMX}$

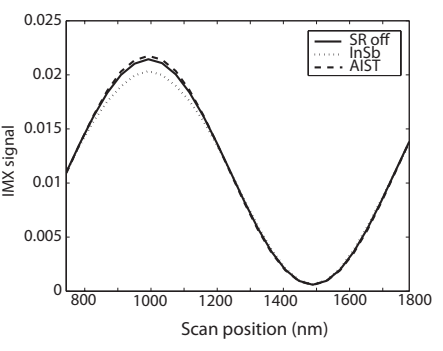

(b) Flat media IMX

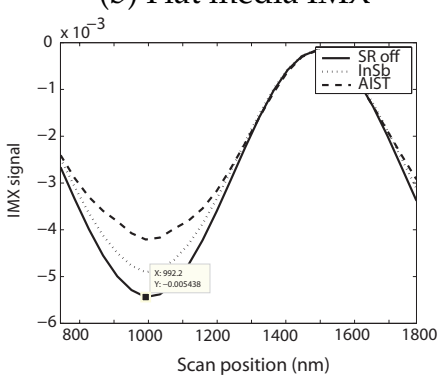

(d) Groove depth $=0.15 \lambda$ IMX
FIG. 12 Readout of a groove-land disc. a) and b) The SMX and IMX signals of a flat disc (groove depth $=0 \lambda$ ) for the InSb, AIST and the non-SRens readout spots. The amount of crosstalk is high at the data mark position. When the groove depth is set to $0.15 \lambda$, the amount of crosstalk is significantly diminished for all readout beams. The land reflectivity used on this calculation is $r_{L}=0.9$ and the marks reflectivity is $r_{M}=0.002$.

disc and in the Figs. 12c and 12d we show the SMX and IMX signals for a groove-land disc, where the groove depth is at the optimal values $(0.15 \lambda)$. The maximum amount of SMX and IMX crosstalk happens when the readout spot is on the mark (between $750 \mathrm{~nm}$ and $1250 \mathrm{~nm}$ ), due to the presence of another data mark right above in the adjacent track. For a flat media (no grooves) being read with the InSb spot, the SMX at $1000 \mathrm{~nm}$ has the highest value of -0.06277 . The IMX signal computed with the AIST spot, on the other hand, has the higher values. In the presence of groove land structure, for the optimized groove depth, the IMX ans SMX crosstalk levels are significantly reduced for all readout spots. At $1000 \mathrm{~nm}$, for instance, the SMX value for the InSb readout spot is -0.01474 (23\% less), as shown in Fig. 12c. Thus, a crosstalk cancelation scheme based on groove-land disc structure seems to be possible for super resolution readout.

\subsection{Two-beam crosstalk cancellation scheme}

In this subsection, we will investigate the possibility of a twobeam crosstalk cancellation scheme, which takes advantage of the SRens focused spot. As seen in the previous sections, the super resolution layer will mainly modify the central region of the focused spot. It suggests that this modified region will be responsible for reading the high frequency information while the remaining of the focused spot would carry the crosstalk information. Therefore, if one uses the two focused spots, one without the super resolution effect (intensity below the threshold limit), and the other one with SRens, a proper subtraction of the readout signal originated from each spot may cancel the crosstalk, leaving only the high frequencies information on the resulting readout signal. In a sense, two beams crosstalk can- 


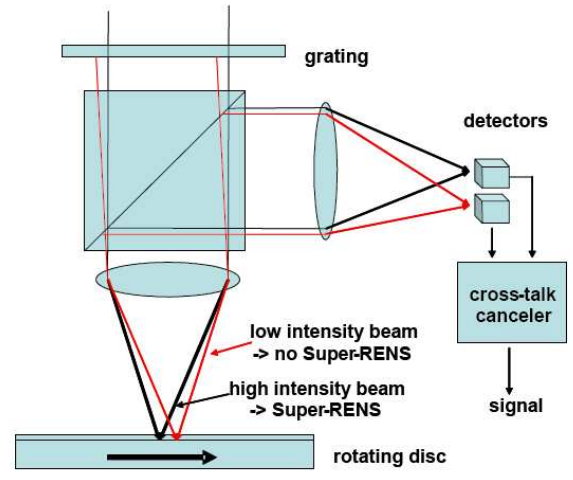

FIG. 13 Schematic of the crosstalk cancelling method using two focused spots. An appropriated subtraction of the two readout signal can cancel the crosstalk.

cellation scheme is preferible over a single beam scheme (see, for instance, Ref. [28]), since the readout signal coming from each spot is clearly identified. However, it requires one additional photo-detector to be used. In our study, we will only consider the crosstalk information coming from data marks on the same scanning track. Thus, adjacent tracks are placed far from the central track and do not influence the readout. A schematic for the two beam crosstalk cancellation approach is shown in Fig. 13.

To illustrate the working principle of this method, we will consider two disc sections depicted in the Figs. 14a and 14b. The first disc section has one small data mark of $250 \mathrm{~nm}$ (beyond the diffraction limit) surrounded by two $500 \mathrm{~nm}$ marks, placed at $125 \mathrm{~nm}$ far from each other. The readout signal of this structure is shown in the Fig. 14c. As seen, none of the three spots (AIST, InSb and SR off) were able to resolve the small data mark, due to the influence of the surrounding 500nm marks. Now, let us consider a two beam subtraction, where the readout signal coming from the beam without SRens effect is subtracted from the signal resulting from the SRens readout. The result is plotted in Fig. 14e for both AIST and InSb readout signals. From the figure, it is clear that a modulated signal occurs for the 250nm data mark, which validates the method. The second disc section considered has three small data marks of $250 \mathrm{~nm}$ surrounded by two pits of $500 \mathrm{~nm}$. As before, the readout signal computed with all three focused spots does not resolve any of the three marks. In the same way, if the two beam subtraction is applied, the small marks become visible in the readout signal. The plots are shown in the Figs. 14d and 14f. It is interesting to note that the super resolution spots could resolve the $250 \mathrm{~nm}$ marks in a periodic structure (see Figs. $7 \mathrm{a}$ and $7 \mathrm{~b}$ ), however, if a $250 \mathrm{~nm}$ mark is surrounded by marks having another frequency, the $250 \mathrm{~nm}$ mark can no longer be resolved by the SRens spots.

\subsection{Adjacent track distance reduction}

As a last analysis, we investigated the possibility of reducing adjacent track distance for the phase change SRens disc. The analyzed disc structure is periodic (data marks length of 500nm) and the adjacent track distance was varied from $750 \mathrm{~nm}$ to $670 \mathrm{~nm}$. To estimate how close the adjacent track can

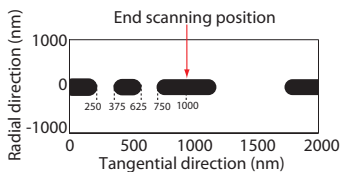

(a) Data structure 1

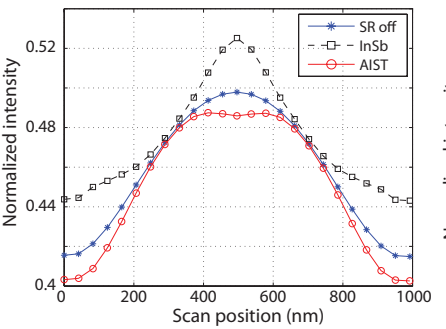

(c) Readout structure 1

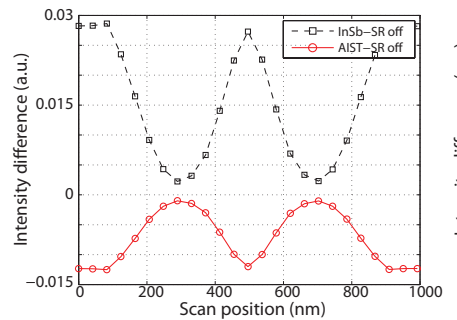

(e) Subtraction 1

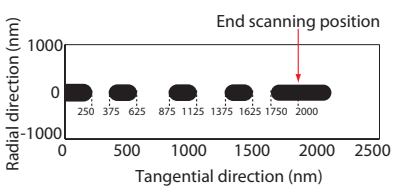

(b) Data structure 2

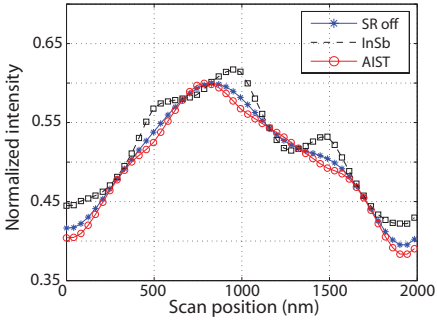

(d) Readout structure 2

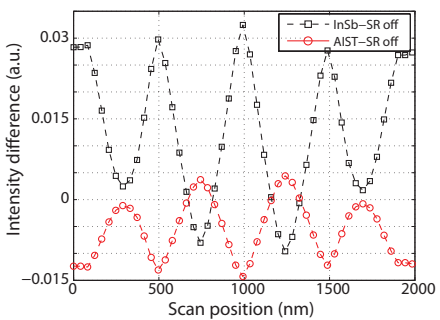

(f) Subtraction 2
FIG. 14 Two-beam crosstalk cancellation. a) and b) show a disc structure containing mark that cannot be resolved by any readout spots [Figs. c) and d)]. However, if the readout signal of the spot without super resolution is subtracted from the SRens signal, the small data marks are resolved.[(e) and (f)]

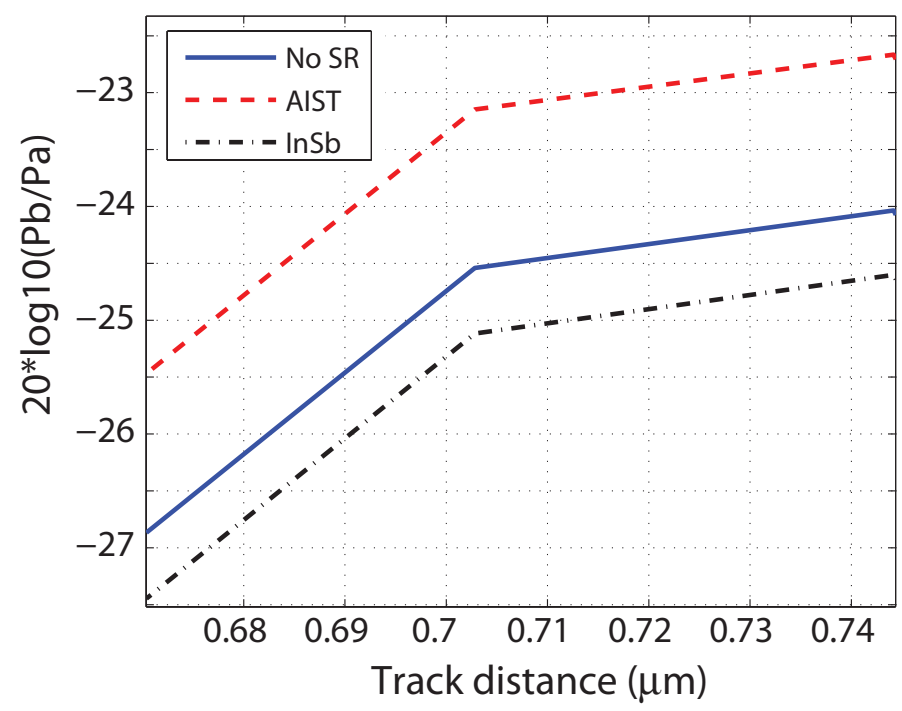

FIG. 15 Estimation of the adjacent track distance reduction for the SRens disc. By keeping as a reference the signal obtained by a focused spot without SRens effect, at a track distance of $740 \mathrm{~nm}$, a decreasing of $7 \%$ of track distance for the AIST based readout is possible. 
be from the reading track, we plotted the ratio $\mathrm{Pa} / \mathrm{Pb}$, where $\mathrm{Pa}$ is the visibility computed after scanning one period of the complete disc section and $\mathrm{Pb}$ is the visibility computed after scanning the same disc section without the central track. The results are displayed in the Fig. 15. Using as reference the signal obtained by the focused spot without SRens effect, at a track distance of 740nm (the standard value for DVD systems), we can estimate that the same amount of crosstalk will be present in the AIST readout signal when the adjacent track is placed at $690 \mathrm{~nm}$. Roughly, this corresponds to a decrease of $7 \%$ of the track distance for the AIST readout. However, no improvements were found for the InSb readout.

\section{Conclusions}

In this work, we have described a scalar readout model for the super resolution near field effect. The SRens effect is incorporated into the focused spot by means of a threshold model. This modified spot is then used to retrieve the readout signal out of several disc structures of interest. It was shown that resolution beyond the diffraction limit is achieved when a periodic structure is read by the SRens spot. The main predictions of this model, among others, include the signal losses at low frequencies that were experimentally observed. Readout signal inversion and the visibility dependence on the pit depth were also observed by the simulation results. Moreover, the focused spot intensity distribution computed with our model resembles those computed rigorously in Ref [24]. The model also helps to understand how the super resolution is achieved, by analysing the diffracted field distribution in the pupil plane. An investigation of the crosstalk effect on the SRens disc was carried out. Results show that the grooveland crosstalk cancellation still holds for the SRens disc and a crosstalk cancellation technique, based on two beam subtraction, can be used to further reduce the crosstalk between adjacent marks. Although not rigorous, our method provides useful insights in the SRens effect, and is a powerful tool to understand the basics of the SRens readout mechanism.

\section{Acknowledgments}

Support to this work by the European Community's Seventh Framework Programme FP7-ICT-2007-2 Surpass Project is gratefully acknowledged. We would like to thank Christophe Fery for his precious help and the team of CEA Leti-Minatec, France for the parameters and discussions.

\section{References}

[1] J. Tominaga, T. Nakano, and N. Atoda, "An approach for recording and readout beyond the diffraction limit with an Sb thin film", Appl. Phys. Lett. 73, 2078 (1998).

[2] J. Tominaga, H. Fuji, A. Sato, T. Nakano, and N. Atoda, "The Characteristics and the Potential of Super Resolution Near-Field Structure", Jpn. J. Appl. Phys. 39, 957 (2000).

[3] J. Tominaga, H. Fuji, A. Sato, T. Nakano, T. Fukaya, and N. Atoda, "The Near-Field Super-Resolution Properties of an Antimony Thin Film", Jpn. J. Appl. Phys. 37, L1323 (1998).
[4] T. Shintani, M. Terao, H. Yamamoto, and T. Naito, "A New SuperResolution Film Applicable to Read-Only and Rewritable Optical Disks", Jpn. J. Appl. Phys. 38, 1656 (1999).

[5] D. Rong Ou, J. Zhu, and J. Hao Zhao, "Approach for imaging optical super-resolution based on Sb films", Appl. Phys. Lett. 82, 1521 (2003).

[6] M. Kuwahara, T. Shima, A. Kolobov, and J. Tominaga, "Thermal Origin of Readout Mechanism of Light-Scattering Super-Resolution Near-Field Structure Disk", Jpn. J. Appl. Phys. 43, L8 (2004).

[7] T. Shima, M. Kuwahara, T. Fukaya, T. Nakano, and J. Tominaga, "Super-Resolutional Readout Disk with Metal-Free Phthalocyanine Recording Layer", Jpn. J. Appl. Phys. 43, L88 (2004).

[8] Y. Yamakawa, K. Kurihara, M. Kuwahara, T. Shima, T. Nakano, and J. Tominaga, “Optical Disc Simulation Program Unified by Electromagnetic and Thermal Distributions", Jpn. J. Appl. Phys. 45, 1463 (2006).

[9] J. Pichon, R. Anciant, J. M. Bruneau, B. Hyot, S. Gidon, M. F. Armand, and L. Poupinet, Multiphysics simulation of superresolution BD ROM optical disk readout, (Optical Data Storage, SPIE, 2006).

[10] J. S. Kim, K. Kwak, and C.-Y. You, "Signal Modulation of Super Read Only Memory with Thermally Activated Aperture Model", Jpn. J. Appl. Phys. 47, 5845 (2008).

[11] A. C. Assafrao, S. F. Pereira, H. P. Urbach, C. Fery, L. von Riewel, and S. Knappmann, "A numerical model for superresolution effect in optical discs", (SPIE, 2010)

[12] A. Fukumoto, and S. Kubota, "Superresolution of Optical Disks Using a Small Aperture", Jpn. J. Appl. Phys. 31, 529 (1992).

[13] T. Ariyoshi, T. Shimano, T. Shintani, and M. Terao, "Read-Out Signal Simulation of an Optical Disk Having an Oxide Super-Resolution Film", Jpn. J. Appl. Phys. 39, 4013 (2000).

[14] J. K. Lee, J. H. Kim, C. S. Chung, I. 0. Hwang, K. H. Jung, and H. K. Kim, "Method and Apparatus for Reproducing Data of Super Resolution Near Field Read-Only Memory disc", U.S. Patent 20060002281 (2006).

[15] H. H. Hophins, "Diffraction theory of laser read-out systems for optical video discs", J. Opt. Soc. Am. 69, 4 (1979).

[16] J. Braat, Readout of Optical discs, Principles of Optical discs, (Adam Hilger, Bristol, UK, 1985).

[17] V. B. Jipson, and C. C. Williams, "Two-dimensional modeling of an optical disk readout", Appl. Optics 14, 2202 (1983).

[18] T. D. Milster, "New Way to Describe Diffraction From Optical Disks", Appl. Optics 37, 6878 (1998).

[19] R. E. Simpson, P. Fons, X. Wang, A. V. Kolobov, T. Fukaya, and J. Tominaga, "Non-melting super-resolution near-field apertures in Sb-Te alloys", Appl. Phys. Lett. 97, 161906 (2010).

[20] Thomson-Villingen, Personal Communication, (Delft, 2010)

[21] M. Kuwahara, 0. Suzuki, N. Taketoshi, Y. Yamakawa, T. Yagi, P. Fons, K. Tsutsumi, M. Suzuki, T. Fukaya, J. Tominaga, and T. Baba, "Measurements of Temperature Dependence of Optical and Thermal Properties of Optical Disk Materials", Jpn. J. Appl. Phys. 45, 1419 (2006).

[22] S. Ohkubo, K. Aoki, and D. Eto, "Temperature dependence of optical constants for InSb films including molten phases", Appl. Phys. Lett. 92, 011919 (2008).

[23] G. Pilard, C. Féry, L. Pacearescu, H. Hoelzemann, and S. Knappmann, "Study of Super-Resolution Read-Only-Memory Disk with a Semiconducting or Chalcogenide Mask Layer", Jpn. J. Appl. Phys. 
48, 03 A064 (2009).

[24] A. C. Assafrao, S. F. Pereira, and H. P. Urbach, "On the Focused Field Embedded in a Super-Rens Medium", Jpn. J. Appl. Phys. 50, 102206 (2011).

[25] J. Tominaga, and T. Nakano, Optical Near Field Recording, (Springer, Heidelberg, 2004).

[26] T. D. Milster, and R. S. Upton, "Fundamental Principles of Crosstalk in Optical Data Storage", Jpn. J. Appl. Phys. 38, 1608 (1999).
[27] N. Miyagawa, Y. Gotoh, E. Ohno, K. Nishiuchi, and N. Akahira, "Land and Groove Recording for High Track Density on PhaseChange Optical Disks", Jpn. J. Appl. Phys. 32, 5324 (1993).

[28] H. Minemura, Y. Anzai, S. Eto, J. Ushiyama, and T. Shintani, "Novel Signal Processing Method for Super-Resolution Discs" in Proceedings on Optical Data Storage, (Optical Society of America, Portland, Oregon, 2007). 